\title{
Laparoscopic Truncal Vagotomy and Gatrojejunostomy for Pyloric Stenosis
}

\author{
Jung-Wook Suh, M.D. ', Ye Seob Jee, M.D., Ph.D. ${ }^{1,2}$ \\ Department of Surgery, 'Dankook University Hospital, ${ }^{2}$ Dankook University School of Medicine, Cheonan, Korea
}

\begin{abstract}
Purpose: Peptic ulcer disease (PUD) remains one of the most prevalent gastrointestinal diseases and an important target for surgical treatment. Laparoscopy applies to most surgical procedures; however its use in elective peptic ulcer surgery, particularly in cases of pyloric stenosis, has not been popular. The aim of this study was to describe the role of laparoscopic surgery and an easily performed procedure for pyloric stenosis. We accordingly performed laparoscopic truncal vagotomy with gastrojejunostomy in 10 consecutive patients with pyloric stenosis.
\end{abstract}

Methods: Data were collected prospectively from all patients who underwent laparoscopic truncal vagotomy with gastrojejunostomy from August 2009 to May 2014 and reviewed retrospectively.

Results: A total of 10 patients underwent laparoscopic trucal vagotomy with gastrojejunostomy for peptic ulcer obstruction from August 2009 to May 2014 in $\bigcirc \bigcirc$ university hospital. The mean age was $62.6( \pm 16.4)$ years old and mean BMI was $19.3( \pm 2.5) \mathrm{kg} / \mathrm{m}^{2}$. There were no conversions to open surgery and no occurrence of intra-operative complications. The mean operation time was 107 (90 130) minutes and blood loss was $<20 \mathrm{ml}$. Oral feeding was permitted for most patients on day 3 post operatively after upper gastrointestinal series to confirm no leakage or passage disturbance. The mean hospital stay was 7.3 days, the mean follow up duration was $19.8( \pm 17.2)$ months, and there was no mortality related to the operation.

Conclusion: Laparoscopic truncal vagotomy and gastrojejunostomy was a good, easily performed surgical choice for patients with duodenal ulcer stricture.

Keywords: Duodenal ulcer, Vagotomy, Laparoscopy, Gastroenterostomy

This is an Open Access article distributed under the terms of the Creative Commons Attribution Non-Commercial License (http:// creativecommons.org/licenses/by-nc/4.0/) which permits unrestricted non-commercial use, distribution, and reproduction in any medium, provided the original work is properly cited.
Received January 27, 2015

Revised 1st March 9, 2015

2nd March 28, 2015

Accepted April 20, 2015

Corresponding author

Ye Seob Jee

Department of Surgery, Dankook

University Hospital, Dankook

University School of Medicine, 119,

Dandae-ro, Dongnam-gu, Cheonan

330-714, Korea

Tel: +82-41-550-3195

Fax: +82-41-550-3912

E-mail: ysjee@dkuh.co.kr

\section{INTRODUCTION}

The prevalence of peptic ulcer disease (PUD) has decreased dramatically because of development of medications such as H2-blockers and proton pump inhibitors (PPI), as well as recognition of the pathophysiology of Helicobacter pylori and its eradication. ${ }^{1-4}$ However, PUD remains one of the most prevalent gastrointestinal diseases and an important target for surgical treatment. ${ }^{5}$ Surgical treatment has also been changed. In the past, vagotomy, antrectomy or drainage was the most common elective, or emergency procedure. Recently, conventional or laparoscopic primary repair of perforated peptic ulcer and over-sewing bleeding vessels for bleeding peptic ulcer are most common procedures. On the other hand, elective surgery including vagotomy and drainage procedure, or antrectomy have greatly decreased. ${ }^{1}$ Today, the majority of practicing general surgeons have at best, only a modest number of elective peptic ulcer surgery cases. ${ }^{6}$ While laparoscopic surgery was popular for most surgical procedures including perforated peptic ulcer, laparoscopy for 
elective peptic ulcer surgery, especially pyloric stenosis, was unpopular. The reason was that the cases were too few to allow sufficient prior experience of open truncal vagotomy for laparoscopic surgery, especially among young surgeons despite their preference for laparoscopic surgery. Generally, vagotomy with gastrojejunostomy is recommended for patient with pyloric stenosis. ${ }^{7}$ We performed laparoscopic truncal vagotomy with gastrojejunostomy in 10 consecutive patients with pyloric stenosis. The aim of this study was introduction of personal experience for laparoscopy truncal vagotomy with gastrojejunostomy and additionally, finding the role of laparoscopic surgery for pyloric stenosis.

\section{MATERIALS AND METHODS}

\section{Patients and methods}

Data was prospectively collected from all patients who underwent laparoscopic truncal vagotomy with gastrojejunostomy, from August 2009 to May 2014 in Dankook University Hospital, Korea, and reviewed retrospectively. All patients had symptoms of gastric outlet obstruction including vomiting, weight loss. Stenosis was confirmed by esophagogastroscopy wherein passage of the scope to the duodenum through the pylorus was not possible. Biopsy was taken for differential diagnosis of malignant ulcer. The biopsied specimen was also examined for Helicobacter pylori infection. The upper gastrointestinal series and computed tomography (CT) were additionally performed. Initially, patients were on medical treatment with PPI and eradication of Helicobacter pylori for several weeks. Nevertheless, we decided on surgical treatment in non-responders to medical treatment and eradication of Helicobacter pylori; such patients were symptomatic for gastric outlet obstruction and had no improvement of stenosis on esophagogatroscopy. Malnutrition and electrolyte imbalance were improved through total parenteral nutrition (TPN). Nasogastric tube was inserted to decompress and reduce edema of the gastric wall and to prevent preoperative aspiration.

\section{Surgical procedure}

The operative procedure of laparoscopic truncal vagotomy with gastrojejunostomy proceeded as described. The patient was placed supine in the reverse Trendelenburg position with the surgeon on the right side of the patient. A total 3 of 4 ports were used ( 3 ports were used when the esophagophrenic junction was well visualized without traction): one $10 \mathrm{~mm}$

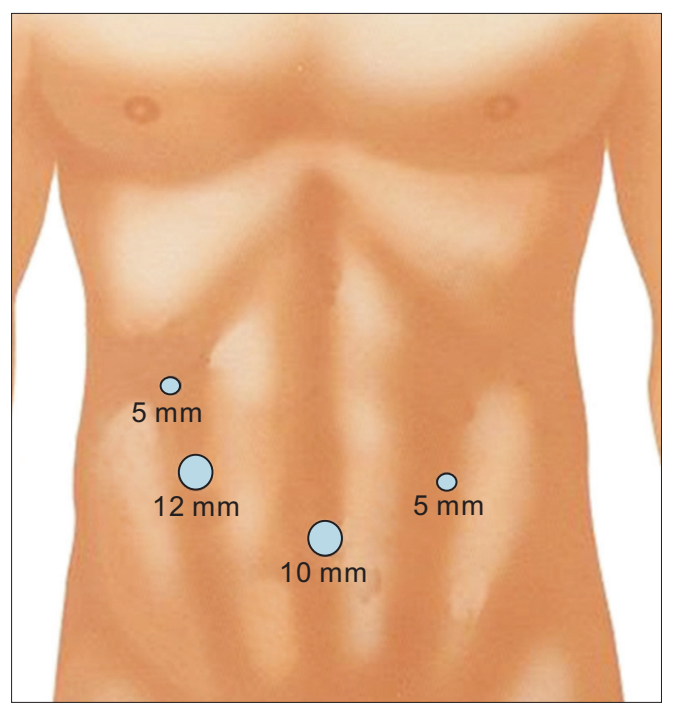

Fig. 1. Tocar palcement for laparoscopic truncal vagotomy with gastrojejunostomy.
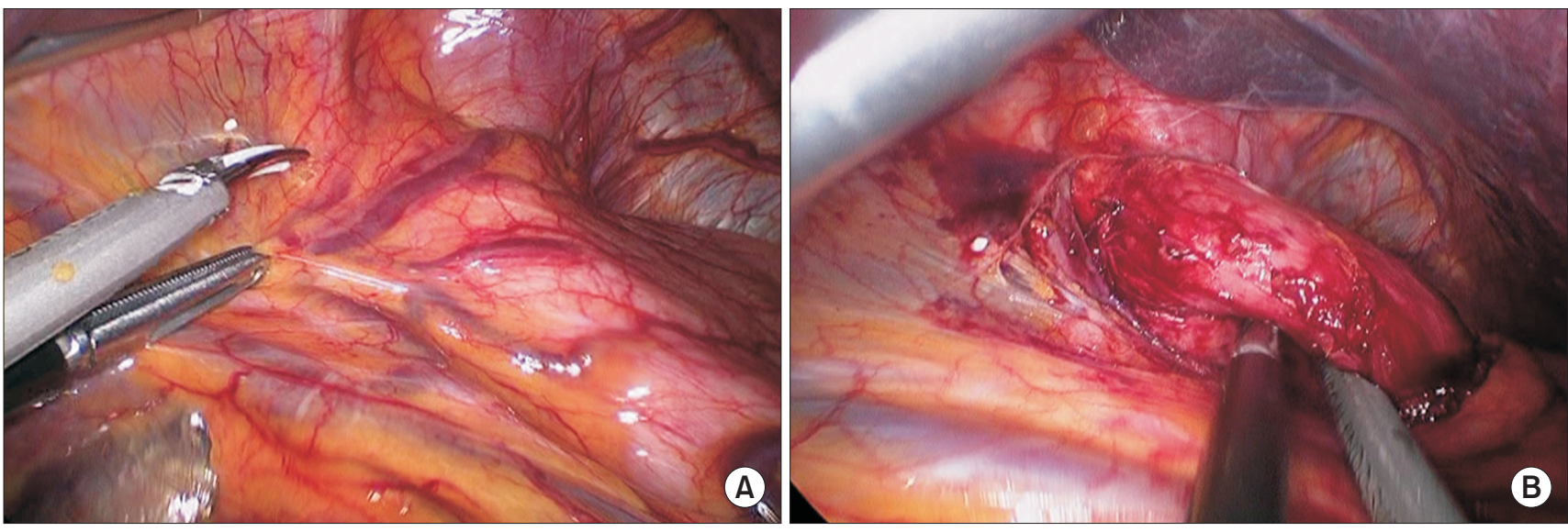

Fig. 2. (A) The hepatogastric ligament was dissected medially with ultrasonic shear. (B) After fully dissected, the esophagus was lifted up and down. 



Fig. 3. (A) The anterior vagus nerve (arrow) was identified and anterior vagotomy was performed with ultrasonic shear. (B) The posterior vagus (arrow) nerve was identified.

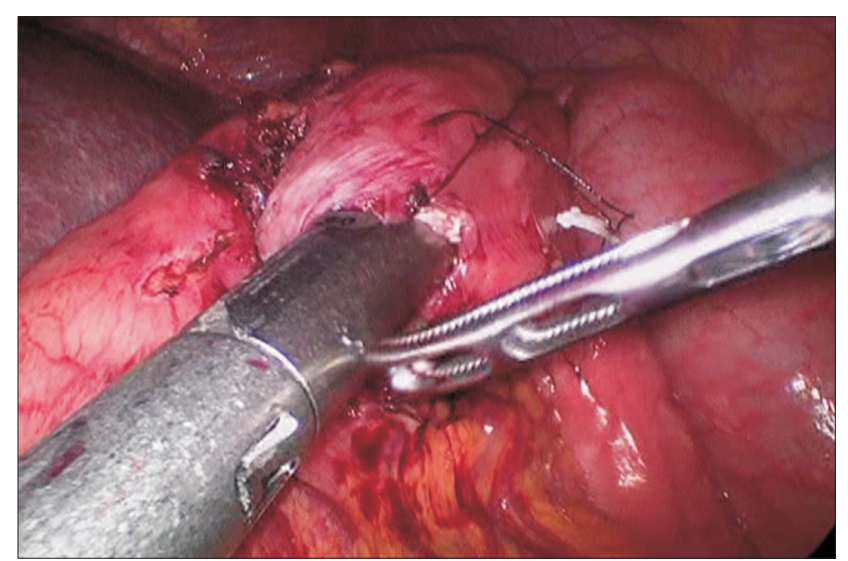

Fig. 4. The side to side gastrojejunostomy was done with a $60 \mathrm{~mm}$ endo linear cutter stapler.

port at the umbilicus for the scope, one $12 \mathrm{~mm}$ and one $5 \mathrm{~mm}$ port at the right midcostal for working port (more cephalomedially when trunk of patient was big), and one 5 $\mathrm{mm}$ port at the left anterior axillary line for liver retraction (Fig. 1). Firstly, the liver was retracted upwardly by gasper forceps or snake retractor to expose the esophagogastric junction. The hepatogastric ligament (lesser omentum) was opened and dissection continued medially to the divide in the phrenoesophageal ligament and extended laterally to ensure mobilization of gastric fundus. The esophagus was lifted up and down and divide from both crus muscle with ultrasonic shears (Fig. 2).

We could then identify the anterior and posterior vagus nerve after the esophagus was free and retracted caudally. Truncal vagotomy was completed using ultrasonic shears without a clip for hemostasis (Fig. 3). The proximal jejunum was pulled up to great curvature and lower body of stomach without tension from Treiz ligament in cases of gastrojejunostomy. The 2 seromuscular suture was done for anchoring the proximal jejunum to stomach. The stomach and jejunum were opened with ultrasonic shears; a $60 \mathrm{~mm}$ sized endo- linear cutter stapler was inserted into the lumen and stapling was done (Fig. 4). We aspirated the gastric juice and checked the gasatrojejunostomy lumen for bleeding and any failure of the stapling line. The common entry hole was closed by intracorporeal continuous suture using a 3-0 polyglycolic suture with seromuscular retention suture using $3-\mathrm{O}$ black silk or endo GIA stapler.

\section{RESULTS}

Total 10 patients underwent laparoscopic trucal vagotomy with gastrojejunostomy for peptic ulcer obstruction, from August 2009 to May 2014 in the $\bigcirc \bigcirc$ university hospital. Table 1. shows patient's demographic and postoperative results. The mean age and BMI were $62.6( \pm 16.4)$ years old and $19.3( \pm$ 2.5) $\mathrm{kg} / \mathrm{m}^{2}$, respectively. There were no conversions to open surgery or intra-operative complications. The mean operation time was 107 (90-130) minutes and blood loss was $<20 \mathrm{ml}$. Most patients were permitted oral feeding at day 3 post operatively after upper gastrointestinal series to confirm no leakage or passage disturbance. The mean hospital stay was 7.3 days. The mean follow up duration was $19.8( \pm 17.2)$ months and there were no mortalities related to the operation. There were 2 postoperative complications and all cases had delayed gastric emptying post-operatively. All patients improved after conservative care. There was no recurrence of related peptic ulcer disease during the follow up period. However, many patients had a bile reflux gastritis through routine follow up esophagogastroscopy. 
Table 1. Patient's demographic and postoperative results

\begin{tabular}{lc}
\hline & Patients $(\mathbf{n}=10)$ \\
\hline Mean (SD) age & $62.6( \pm 16.4)$ \\
Sex & 9 \\
Male & 1 \\
Female & \\
ASA classification & 3 \\
I & 4 \\
II & 3 \\
III & 0 \\
IV & $19.3( \pm 2.5)$ \\
Mean (SD) BMI (Kg/m²) & 107 (90 130) \\
Operation duration (minutes), mean (range) & $3(2 \sim 4)$ \\
Resume diet day, mean (range) & $7.8(6 \sim 13)$ \\
Post op hospital stay, mean (range) & 2 (Delayed gastric emptying) \\
Post op complication & None \\
Post op. mortality & $19.8( \pm 17.2)$ \\
Mean follow up months (SD) & None \\
\hline Ulcer recurrence &
\end{tabular}

\section{DISCUSSION}

The incidence and prevalence of peptic ulcer disease have dramatically decreased since the past 4 decades, with a concomittant decrease in the incidence of elective surgery including intractability and obstruction. ${ }^{1-5}$ However the incidence of emergent surgeries for perforation and bleeding have remained relatively constant or even increased. Most operations for peptic ulcer disease are for perforation and bleeding, currently. Many surgeons might prefer simple closure and suture ligation bleeding vessels than definite ulcer surgery because of the development of medical therapy. Therefore, the experience of the general surgeon in an era of decreased elective surgery is insufficient for definite ulcer surgery. However, even now some patients require definite ulcer operation in cases of failure of medical treatment; hence young general surgeons have to learn about acid reducing surgery including open or laparoscopic surgery.

We thought that performing and learning laparoscopic trucal vagotomy was easier than open surgery. During open surgery, the assistant's view of vagus nerves was usually obstructed by the left lobe of the liver and the operator's hand. Furthermore, tactile sense was important to find the vagus nerve and especially the posterior vagus nerve, during open trucal vagotomy. Finding the vagus nerve was easier during laparoscopic surgery, than open surgery. Additionally, the assistant could see and learn the vagotomy procedure. Fig. 2 showed that the left lobe of liver was easily retracted, therefore the view of the vagus nerve by the laparoscopy camera system was more delicately supported than by the direct view of open surgery. The surgeon could review and study the surgical procedure post-surgery by watching recorded videos repeatedly. Recently, because of developing laparoscopic stapling device and technique, entero-enterostomy including gastrojejunostomy, can be performed easily and safely. Fig, 4 showed the laparoscopic gastrojejunostomy. The modern surgeon is likely to be well experienced in this procedure because it has already been performed skillfully in the field of several variable gastrointestinal surgeries, including gastric and bariatric surgery.

The limitation of this study was that it was not a comparative study with open surgery. However we believed the early results of laparoscopy surgery such as laparoscopic cholecystectomy were more favorable than open surgery in terms of postoperative pain, shorter hospital stay, pulmonary complication and cosmetics. Additionally, our results showed that there were no post operative complications, such as atelectasis and wound complication.

There were only $1 \sim 2$ case per year of peptic ulcer stricture in our hospital. On the other hand, the cases of emergency operation especially, laparoscopic primary repair was $>50$ cases per year. We thought that this trend led to a decrease in elective and definite ulcer surgery similar to another hospital; therefore, the experience of definite ulcer surgery i.e. vagotomy and drainage procedure was insufficient especially in young surgeons. In this study, we observed laparoscopy truncal vagotomy with gastrojejunostomy could be performed easily and the results were acceptable. Laparoscopy truncal vagotomy with gastrojejunostomy was thus a good choice for peptic ulcer with pyloric stenosis, given the education of young surgeons and improving early surgical results in the era of decreasing definite ulcer surgery.

\section{REFERENCES}

1) Stabile BE, Passaro E, Jr. Duodenal ulcer: a disease in evolution. Curr Probl Surg 1984;21:1-79.

2) Isenberg JI. The impact of H2-receptor antagonists on the complications, morbidity and mortality of peptic ulcer disease. Aliment Pharmacol Ther 1987;1 Suppl 1:447s-454s.

3) McKay AJ, McArdle CS. Cimetidine and perforated peptic ulcer. Br J Surg 1982;69:319-320.

4) Primatesta $P$, Goldacre MJ, Seagroatt V. Changing patterns in the epidemiology and hospital care of peptic ulcer. Int J Epidemiol 
1994;23:1206-1217.

5) McConnell DB, Baba GC, Deveney CW. Changes in surgical treatment of peptic ulcer disease within a veterans hospital in the 1970s and the 1980s. Arch Surg 1989;124:1164-1167.

6) Harbison SP, Dempsey DT. Peptic ulcer disease. Curr Probl Surg
2005;42:346-454.

7) Jr. Townsend CM, Beauchamp RD, Evers BM, Mattox KL. Sabiston Textbook of Surgery: The Biological Basis of Modern Surgical Practice. 18th ed. Philadelphia: W.B Saunders; 2008. 